\title{
Wavelets versus resels in the context of fMRI: establishing the link with SPM
}

\author{
Dimitri Van De Ville, Thiery Blu, Michael Unser \\ Biomedical Imaging Group, Swiss Federal Institute of Technology Lausanne, \\ Bâtiment Microtechnique, CH-1015 Lausanne EPFL, Switzerland
}

\begin{abstract}
Statistical Parametric Mapping (SPM) is a widely deployed tool for detecting and analyzing brain activity from fMRI data. One of SPM's main features is smoothing the data by a Gaussian filter to increase the SNR. The subsequent statistical inference is based on the continuous Gaussian random field theory. Since the remaining spatial resolution has deteriorated due to smoothing, SPM introduces the concept of "resels" (resolution elements) or spatial information-containing cells. The number of resels turns out to be inversely proportional to the size of the Gaussian smoother.

Detection the activation signal in fMRI data can also be done by a wavelet approach: after computing the spatial wavelet transform, a straightforward coefficient-wise statistical test is applied to detect activated wavelet coefficients. In this paper, we establish the link between SPM and the wavelet approach based on two observations. First, the (iterated) lowpass analysis filter of the discrete wavelet transform can be chosen to closely resemble SPM's Gaussian filter. Second, the subsampling scheme provides us with a natural way to define the number of resels; i.e., the number of coefficients in the lowpass subband of the wavelet decomposition. Using this connection, we can obtain the degree of the splines of the wavelet transform that makes it equivalent to SPM's method. We show results for two particularly attractive biorthogonal wavelet transforms for this task; i.e., 3D fractional-spline wavelets and $2 \mathrm{D}+\mathrm{Z}$ fractional quincunx wavelets. The activation patterns are comparable to SPM's.
\end{abstract}

Keywords: fMRI data analysis, discrete wavelet transform, statistical testing, Gaussian smoothing

\section{INTRODUCTION}

Functional magnetic resonance imaging (fMRI) allows us to non-invasively observe brain activity. The neuronal activity can be linked to physiological changes (such as blood oxygenation and blood flow) which, in turn, induce a weak and noisy contrast signal in the acquired images. ${ }^{1-3}$

The widely deployed and recognized method for performing the analysis of fMRI data is the Statistical Parametric Map (SPM). ${ }^{4}$ Since many years, collaborators at the Wellcome Department of Cognitive Neurology (UCL, London, UK) make the corresponding software package freely available to the neurology community. One of its main features is to prefilter the data by a Gaussian smoothing filter to increase SNR. The subsequent statistical inference uses the theory of continuous Gaussian random fields.

The analysis of fMRI data using a spatial wavelet transform was first proposed by Ruttimann et al. ${ }^{5}$ The main idea is not to prefilter the data, but to use the wavelet transform to improve the detection results. The statistical inference is performed in the wavelet domain by a simple coefficient-wise t-test. ${ }^{6}$ Compared to previous publications using this wavelet approach, ${ }^{5-8}$ the present paper introduces some improvements

- The use of two types of 3D wavelet transforms: the 3D separable fractional-spline wavelets, and a $2 \mathrm{D}+\mathrm{Z}$ quincunx wavelet transform.

- The wavelet coefficients time-series are modelled by the general linear model (GLM), a versatile way to setup the experiment and to incorporate the effect of the hemodynamic response function. ${ }^{9}$

In the following section, we will further highlight the most important characteristics of both SPM and the wavelet approach. Next, we elaborate a remarkable link between both. This link is based on a particular choice of basis functions at the analysis side of the wavelet transform.

Send correspondence to Dimitri Van De Ville, E-mail: Dimitri.VanDeVille@epfl.ch, Telephone: +41 216935142 


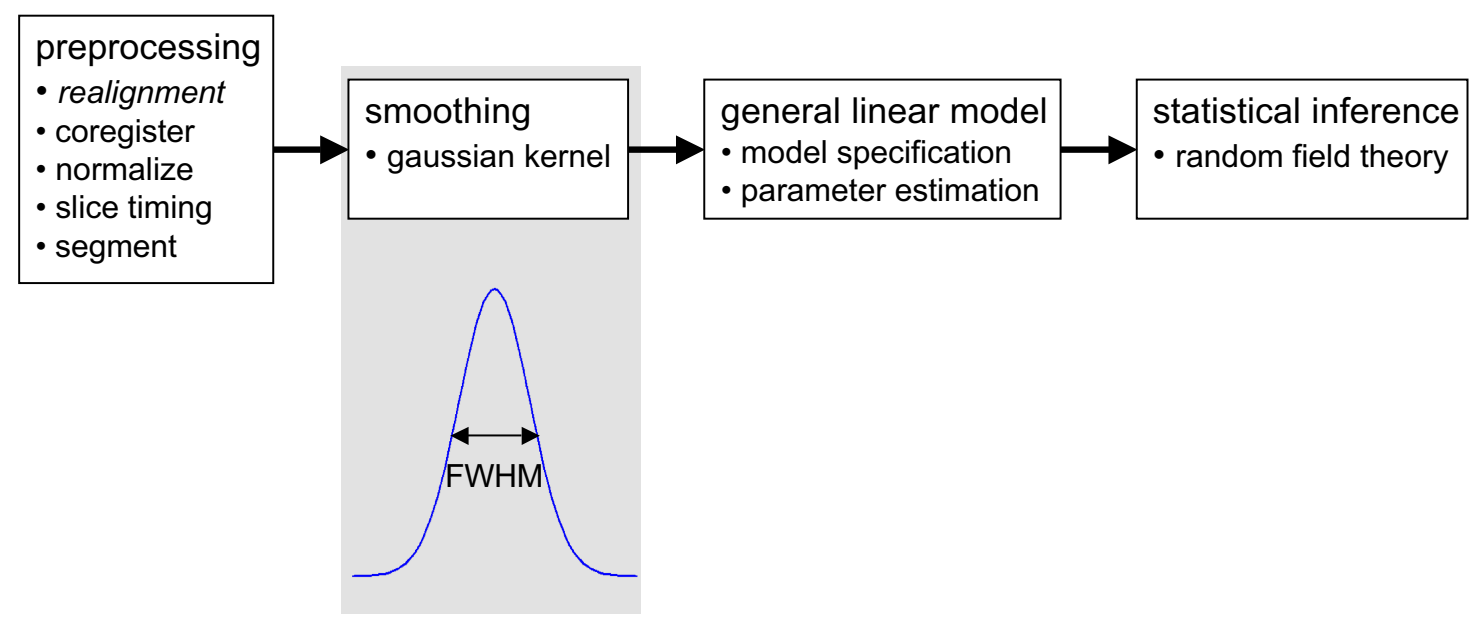

Figure 1. SPM's main features relevant to this paper: processing raw data up to statistical inference.

\section{METHODS TO ANALYSE FMRI DATA}

\subsection{Statistical Parametric Map}

We start by giving an overview of the features of SPM (see Fig. 1) that are relevant to this paper; i.e., processing the raw data up to statistical inference. The data are first aligned before getting smoothed by a Gaussian filter. This filter is characterized by its FWHM (full width at half maximum). Next, the general linear model (GLM) is applied voxel-wise. The time-series of each voxel are modelled by a so-called design matrix $\mathbf{X}$ and a residual gaussian error e:

$$
\mathbf{v}=\mathbf{X y}+\mathbf{e}
$$

The maximum likelihood estimate $\hat{\mathbf{y}}$ of the model parameters is found by a least-squares solution*. Next, the parameter selection is done by a contrast vector $\mathbf{c}$, which selects or compares one or multiple parameter values. The final step is the statistical inference which takes into account the dependencies introduced by the Gaussian filtering. To this end, SPM relies on the theory of continuous random Gaussian fields. An important concept in this context is that of a resel (resolution element or information-containing cell). The average number of resels available in the data after smoothing is defined as

$$
\# \text { resels }=\frac{V}{\Pi_{i=1}^{3} \mathrm{FWHM}_{i}},
$$

where $V$ is the number of voxels and $\mathrm{FWHM}_{i}$ is the Gaussian width for the $i$-th dimension.

\subsection{Wavelet Approach}

In Fig. 2, we give an overview of the wavelet-based approach. After alignment of the volumes, one first computes the spatial non-redundant discrete wavelet transform (DWT). Since the data is decomposed in a one-to-one fashion, the high-frequency information (contained in the wavelet subbands) is not lost and can still influence the results. Next, the general linear model is applied to the time-series of each wavelet coefficient. The activation of a coefficient is detected by a simple t-test in the wavelet domain, using a conservative Bonferroni-correction for multiple testing. Finally, the pattern is localized by the inverse wavelet transform of the thresholded coefficient map.

The discrete wavelet transform is implemented through an iterated filterbank, ${ }^{10}$ whose analysis and synthesis parts are depicted in Fig. 3. To handle our 3D fMRI data, we propose the use of two types of wavelet families: the separable $3 \mathrm{D}$ fractional-spline wavelets and the $2 \mathrm{D}+\mathrm{Z}$ quincunx wavelets.

\footnotetext{
${ }^{*}$ Here we assume no temporal correlations between the data.
} 


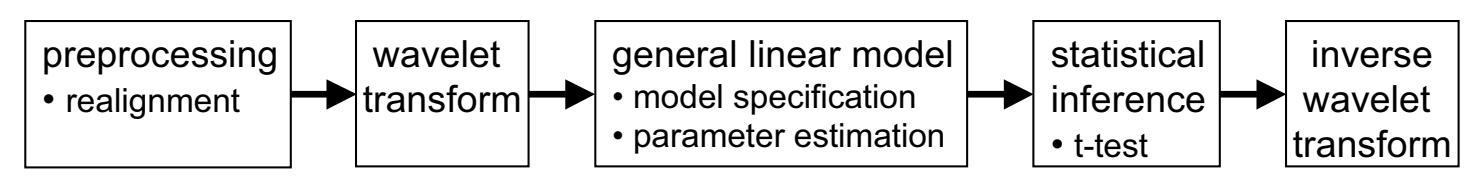

Figure 2. Schematical overview of the wavelet approach.

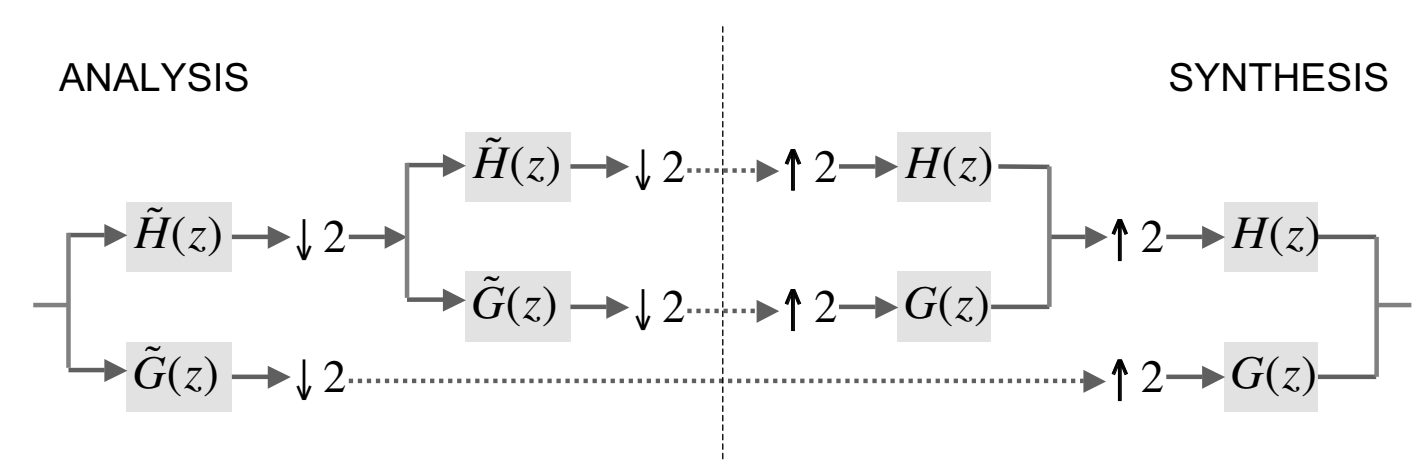

Figure 3. The principle of the 1D discrete wavelet transform. The analysis side decomposes the signal into a lowpass (filter $\tilde{H}(z)$ ) and highpass (filter $\tilde{G}(z)$ ) subband, followed by subsampling. Next, the lowpass subband is iterated. The synthesis side reconstructs the original signal.

\subsubsection{Separable 3D fractional-spline wavelets}

Fractional splines are an elegant generalization of classical cardinal splines. ${ }^{11,12}$ This class of splines has two parameters: the degree is a continuously-varying parameter $\alpha>-1 / 2$, and the shift parameter $\tau$. In this paper, we will consider only the symmetric flavor $\tau=0$ with the following expression in the Fourier domain

$$
\hat{\beta}^{\alpha}(\omega)=\left|\frac{1-\exp (-j \omega)}{j \omega}\right|^{\alpha+1} .
$$

These functions can be used as scaling functions for a wavelet transform. Their two-scale relationship is given by the scaling filter $B^{\alpha}(z)=\sqrt{2}\left|\frac{1+z^{-1}}{2}\right|^{\alpha+1}$. Both orthogonal and biorthogonal transforms can be constructed. Of particular importance for the remaining part of this paper are the so-called "dual" filters. Here, the analysis part consists of the pure B-spline filter (and some corresponding highpass filter for the wavelet channel), while the synthesis part consists of the dual part, which is less well localized. The fractional-spline wavelet transforms are implemented using the Fast Fourier Transform; this implementation (for Matlab) is freely available at http://bigwww.epfl.ch/demo/fractsplines/. To handle our 3D fMRI data, we apply the wavelet transform separately for each dimension.

\subsection{2. $2 \mathrm{D}+\mathrm{Z}$ quincunx wavelets}

Another interesting approach is to use the non-separable 2D quincunx scheme for the XY-planes, ${ }^{13}, 14$ combined with a separable transform along the Z-direction. The quincunx subsampling matrix corresponds to

$$
\mathbf{D}=\left[\begin{array}{cc}
1 & 1 \\
1 & -1
\end{array}\right]
$$

and has a determinant of -2 . So each $2 \mathrm{D}$ iteration introduces only two subbands (as opposed to 4 in the case of a $2 \mathrm{D}$ separable transform). 
To derive the $2 \mathrm{D}$ quincunx filter, we start to consider a biorthogonal filter pair where the first one is a fractional iterate of the simplest symmetric refinement filter of order 2 :

$$
Q^{\alpha_{q}}(\exp (j \omega))=\sqrt{2}\left(\frac{2+2 \cos \omega}{4}\right)^{\frac{\alpha_{q}+1}{2}},
$$

and the second is obtained by its $l_{2}$-dual:

$$
\frac{\sqrt{2}(2+2 \cos \omega)^{\frac{\alpha_{q}+1}{2}}}{(2+2 \cos \omega)^{\alpha_{q}+1}+(2-\cos \omega)^{\alpha_{q}+1}} .
$$

These filters define the lowpass branch of a perfect reconstruction filterbank. We construct a $2 \mathrm{D}$ quincunx filterbank by applying the McClellan transform, ${ }^{15}$ which amounts to simply replacing $\cos \omega$ by $\left(\cos \omega_{1}+\cos \omega_{2}\right) / 2$. For the purpose of this paper, we are particularly interested in the biorthogonal transform where the filter $Q^{\alpha_{q}}(\exp (j \omega))$ is placed at the analysis side. The corresponding wavelet filters are obtained by permuting the filters and modulating them.

\section{ESTABLISHING THE LINK}

\subsection{Gaussian smoothing revisited}

Let us consider the biorthogonal wavelet transform with the B-spline at the analysis side. In such a case, the B-spline analysis scaling function associated with the low-pass subband rapidly converges to a Gaussian as the degree increases. ${ }^{16}$ Consequently, one can identify the equivalent FWHM of the lowpass analysis filter for the wavelet transform after $J$ iterations. First, we start by identifying the equivalent FWHM for a 1D fractionalspline wavelet.

1. After one iteration, the lowpass filter corresponding to a B-spline filter of degree $\alpha$, can be approximated as:

$$
\begin{aligned}
B^{\alpha}(\exp (j \omega)) & =\sqrt{2}\left|\frac{1+\exp (-j \omega)}{2}\right|^{\alpha+1} \\
& =\sqrt{2}|\cos (\omega / 2)|^{\alpha+1} \\
& =\sqrt{2} \exp ((\alpha+1) \ln |\cos (\omega / 2)|) \\
& \approx \sqrt{2} \exp \left((\alpha+1) \ln \left(1-\frac{\omega^{2}}{8}\right)\right) \\
& \approx \sqrt{2} \exp \left(-(\alpha+1) \frac{\omega^{2}}{8}\right)
\end{aligned}
$$

2. Using Noble's identity, we obtain the filter $B_{J}^{\alpha}$ corresponding to $J$ iterations as

$$
\begin{aligned}
B_{J}^{\alpha}(\exp (j \omega)) & =B^{\alpha}(\exp (j \omega)) B^{\alpha}(\exp (2 j \omega)) \ldots B^{\alpha}\left(\exp \left(2^{J-1} j \omega\right)\right) \\
& \approx 2^{J / 2} \exp \left(-\frac{\alpha+1}{8}\left(\omega^{2}+4 \omega^{2}+\ldots+4^{J-1} \omega^{2}\right)\right) \\
& \approx 2^{J / 2} \exp \left(-\frac{\alpha+1}{8} \omega^{2} \frac{4^{J}-1}{3}\right)
\end{aligned}
$$

3. The Fourier transform of the Gaussian kernel is given by

$$
\frac{1}{\sigma \sqrt{2 \pi}} \exp \left(-\frac{x^{2}}{2 \sigma^{2}}\right) \longleftrightarrow \exp \left(-\frac{\sigma^{2} \omega^{2}}{2}\right),
$$

and allows us to identify the equivalent FWHM at scale $J$, as

$$
\mathrm{FWHM}=2 \sigma \sqrt{2 \ln (2)}=\sqrt{2 \ln (2)} \sqrt{\alpha+1} \sqrt{\frac{4^{J}-1}{3}} .
$$


For the 2D quincunx case, we can obtain the following result:

1. The lowpass filter $Q^{\alpha_{q}}(\exp (j \omega))$ of degree $\alpha_{q}$ corresponding to the first iteration can be approximated in a similar way as:

$$
\begin{aligned}
Q^{\alpha_{q}}(\exp (j \omega)) & =\sqrt{2}\left(\frac{2+\cos \omega_{1}+\cos \omega_{2}}{4}\right)^{\frac{\alpha_{q}+1}{2}} \\
& =\sqrt{2}\left(\frac{\cos ^{2}\left(\omega_{1} / 2\right)+\cos ^{2}\left(\omega_{2} / 2\right)}{2}\right)^{\frac{\alpha_{q}+1}{2}} \\
& =\sqrt{2} \exp \left(\frac{\alpha_{q}+1}{2} \ln \left(\frac{\cos ^{2}\left(\omega_{1} / 2\right)+\cos ^{2}\left(\omega_{2} / 2\right)}{2}\right)\right) \\
& \approx \sqrt{2} \exp \left(\frac{\alpha_{q}+1}{2} \ln \left(1-\frac{\omega_{1}^{2}}{8}-\frac{\omega_{2}^{2}}{8}\right)\right) \\
& \approx \sqrt{2} \exp \left(-\frac{\alpha_{q}+1}{16}\left(\omega_{1}^{2}+\omega_{2}^{2}\right)\right) .
\end{aligned}
$$

2. Using Noble's identity, once more we obtain the filter $Q_{J_{q}}^{\alpha_{q}}$ corresponding to $J_{q}$ iterations:

$$
\begin{aligned}
Q_{J_{q}}^{\alpha_{q}}(\exp (j \boldsymbol{\omega})) & =Q^{\alpha_{q}}(\exp (j \boldsymbol{\omega})) Q^{\alpha_{q}}\left(\exp \left(j \mathbf{D}^{\mathrm{T}} \boldsymbol{\omega}\right)\right) \ldots Q^{\alpha_{q}}\left(\exp \left(j\left(\mathbf{D}^{\mathrm{T}}\right)^{2^{J_{q}-1}} \boldsymbol{\omega}\right)\right) \\
& \approx 2^{J_{q} / 2} \exp \left(-\frac{\alpha_{q}+1}{16}\left(\omega_{1}^{2}+\omega_{2}^{2}+2 \omega_{1}^{2}+2 \omega_{2}^{2}+\ldots+2^{J_{q}-1} \omega_{1}^{2}+2^{J_{q}-1} \omega_{2}^{2}\right)\right) \\
& \approx 2^{J_{q} / 2} \exp \left(-\frac{\alpha_{q}+1}{16}\left(\omega_{1}^{2}+\omega_{2}^{2}\right)\left(2^{J_{q}}-1\right)\right)
\end{aligned}
$$

3. The equivalent FWHM can now be identified as

$$
\mathrm{FWHM}=\sqrt{\ln (2)} \sqrt{\alpha_{q}+1} \sqrt{2^{J_{q}}-1} .
$$

\subsection{Resels revisited}

Resels or resolution elements are spatial information-containing cells. Initially, that is, before smoothing, the number of resels can be considered equal to the number of voxels. After Gaussian smoothing by SPM, their number is reduced according to Eq. (2). For the wavelet approach, we introduce the number of resels as the number of coefficients left in the lowpass subband.

For the 3D separable fractional-spline wavelet transform, we obtain

$$
\# \text { resels }=\frac{V}{2^{J_{1}+J_{2}+J_{3}}} \Rightarrow \Pi_{i=1}^{3} \mathrm{FWHM}_{i}=2^{J_{1}+J_{2}+J_{3}},
$$

where $J_{1}, J_{2}$, and $J_{3}$ are the number of iterations in each dimension.

For the $2 \mathrm{D}+\mathrm{Z}$ quincunx wavelet transform, we obtain

$$
\# \text { resels }=\frac{V}{2^{J_{q}+J_{z}}} \quad \Rightarrow \quad \Pi_{i=1}^{3} \mathrm{FWHM}_{i}=2^{J_{q}+J_{z}},
$$

where $J_{q}$ and $J_{z}$ are respectively the number of iterations in the XY-plane (quincunx) and the Z-direction.

\subsection{The link in the case of isotropic sampling}

Usually, the FWHM of the Gaussian filter is specified in physical units, e.g., FWHM=6mm. The coefficients of the filter mask are obtained by sampling the Gaussian function at the sampling steps corresponding to the physical voxel units. In the case of isotropic voxels, we have $\mathrm{FWHM}_{i}=\mathrm{FWHM}$ for $i=1,2,3$. By comparing the expressions for the FWHM in Section 3.1 and 3.2, we may deduce a spline degree that has an effect that is equivalent to SPM's Gaussian smoothing. 
(a)

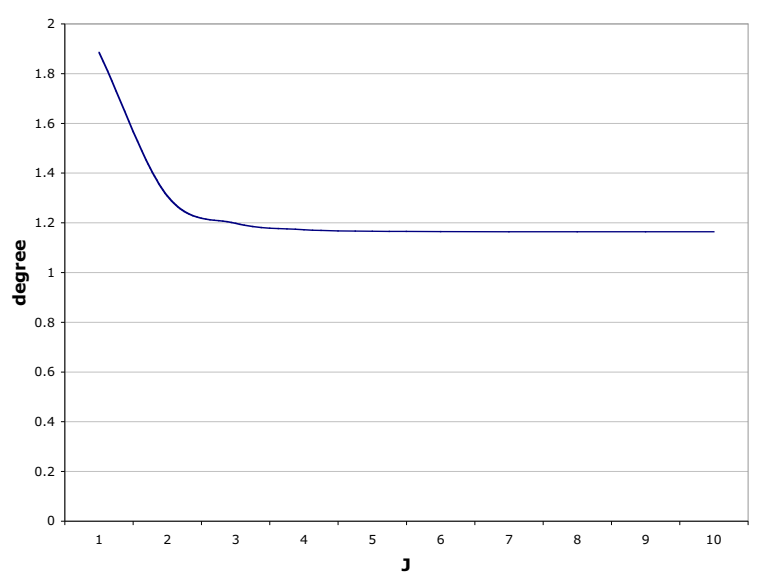

(b)

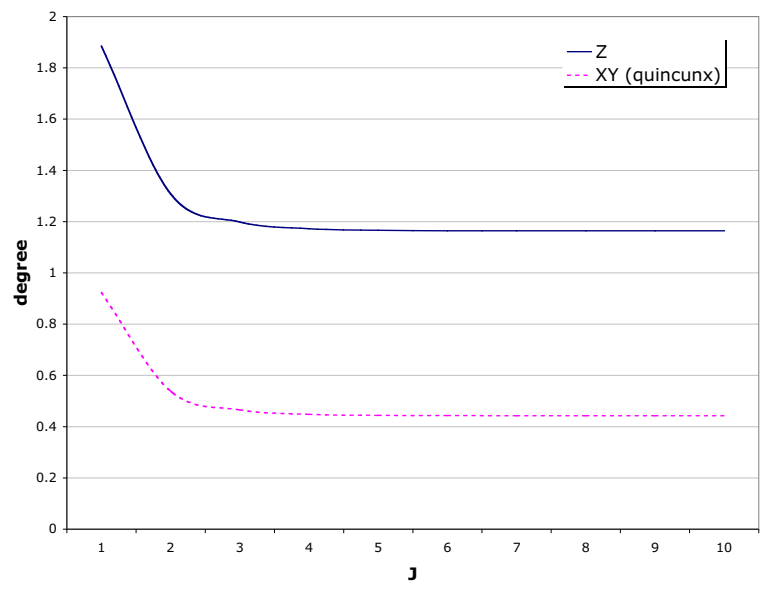

Figure 4. Equivalent degree for the wavelet approach, isotropic case. (a) 3D separable fractional-spline wavelets. (b) $2 \mathrm{D}+\mathrm{Z}$ quincunx wavelets.

\subsubsection{D separable fractional-spline wavelet}

In the case of isotropic sampling, it is reasonable to keep the resel for the wavelet approach isotropic as well. Therefore, we perform the same number of iterations $J_{1}=J_{2}=J_{3}=J$ in each dimension. By equating the expressions for FWHM given by Eqs. (8) and (10), we obtain the following equation for the degree

$$
2^{J}=\sqrt{2 \ln (2)} \sqrt{\alpha+1} \sqrt{\frac{4^{J}-1}{3}} \Rightarrow \alpha=6 \frac{4^{J-1}}{\left(4^{J}-1\right) \ln 2}-1 .
$$

Figure 4 (a) shows the degree $\alpha$ as function of $J$. Interestingly, the equivalent degree converges to $\alpha=1.164$ for $J \rightarrow+\infty$.

\subsection{2. $2 \mathrm{D}+\mathrm{Z}$ quincunx wavelet}

Here too, we want to keep the resel isotropic. However, since the a 2D quincunx iteration downsamples two times slower as a Z iteration, we need to perform twice as much quincunx iterations: $J_{q}=2 J_{z}=2 J$. The degrees can then be obtained as

$$
\begin{gathered}
2^{J}=\sqrt{\ln (2)} \sqrt{\alpha_{q}+1} \sqrt{4^{J}-1} \Rightarrow \alpha_{q}=\frac{4^{J}}{\left(4^{J}-1\right) \ln 2}-1, \\
2^{J}=\sqrt{2 \ln (2)} \sqrt{\alpha_{z}+1} \sqrt{\frac{4^{J}-1}{3}} \Rightarrow \alpha_{z}=6 \frac{4^{J-1}}{\left(4^{J}-1\right) \ln 2}-1 .
\end{gathered}
$$

Figure 4 (b) shows both degrees as function of $J$. Notice that the equivalent degree for the quincunx filter becomes fairly small, which renders the approximation to the Gaussian filter less precise.

\subsection{Non-isotropic sampling}

We now examine the case that is most relevant to fMRI applications; i.e., the Z-direction is sampled coarser than the $\mathrm{X}$ and $\mathrm{Y}$ directions. For example, let the size of the voxels be $u_{x}, u_{y}, u_{z}$ with $u_{x}=u_{y}$ and $u_{z}=\gamma u_{x}$ $(\gamma \geq 1)$. Therefore, we can introduce $\mathrm{FWHM}_{1}=\mathrm{FWHM}_{2}=\mathrm{FWHM}$ and $\mathrm{FWHM}_{3}=\mathrm{FWHM} / \gamma$. 


\subsubsection{D separable fractional spline wavelet}

The corresponding expression for the FWHM, which is obtained from Eqs. (2) and (10), is

$$
\mathrm{FWHM}=\gamma^{1 / 3} 2^{\frac{J_{1}+J_{2}+J_{3}}{3}}
$$

Still, it is reasonable to iterate in each dimension at the same rate (to maintain the resel's dimensions). However, if we simply compute the degrees for $J_{i}=J(i=1,2,3)$, then we observe that $\alpha_{1}=\alpha_{2}$ diverge from $\alpha_{3}$ as $\gamma$ increases. The solution we propose is to pre-iterate $k$ times in the $X$ and $Y$ directions to compensate for the non-isotropy of the filter

$$
J_{1}=J+k, \quad J_{2}=J+k, \quad J_{3}=J,
$$

with $k=\operatorname{round}\left(\log _{2} \gamma\right)$. After $k$ pre-iterations in the $X$ and $Y$ directions, the sampling density of the remaining lowpass subband becomes approximately (exactly when $\gamma$ is a power of 2 ) uniform.

\subsection{2. $2 \mathrm{D}+\mathrm{Z}$ quincunx wavelet}

The same correction needs to be done for the $2 \mathrm{D}+\mathrm{Z}$ quincunx transform. The expression for the FWHM equals

$$
\mathrm{FWHM}=\gamma^{1 / 3} 2^{\frac{J_{q}+J_{z}}{3}},
$$

and the number of iterations can be computed as:

$$
J_{q}=2 J+k, \quad J_{z}=J
$$

with $k=2 \operatorname{round}\left(\log _{2} \gamma\right)$.

\section{EXAMPLE}

We show an example of an fMRI experiment (auditory stimulation) of a block-based paradigm. ${ }^{17}$ The voxels are isotropic $(3 \mathrm{~mm} \times 3 \mathrm{~mm} \times 3 \mathrm{~mm})$. The setup of the design matrix has been done by SPM and incorporates a model for the hemodynamic response function. The same general linear model has been used both by SPM and the wavelet approach. The confidence level has been fixed at $5 \%$. Figure 5 shows the detected activation of a slice at the auditory cortex for the various methods. Notwithstanding the conservative statistical approach of the wavelet method (i.e., Bonferroni-correction for multiple testing), we obtain comparable activation patterns as SPM. The result for $2 \mathrm{D}+\mathrm{Z}$ quincunx with $J=3$ seems to give a worser result. This can be explained by the fact that the degree $\alpha_{q}=0.466$ is small and that the quincunx filter has not yet converged to a Gaussian. The current wavelet approach still needs a rather ad-hoc uniform spatial threshold ${ }^{5}$ to distinguish between activated and non-activated voxels after reconstruction of the thresholded coefficient map. A related disadvantage of the wavelet approach is that the precise statistical meaning (i.e., a confidence measure such as a $t$-value) of the detection is lost once the activation is mapped back in the spatial domain. On the other hand, we believe that the wavelet approach can potentially produce activation maps of higher resolution. For example, the SPM result in Fig. 6 is rather similar to the map we obtain when using coefficients from the lowpass subband only. A full wavelet detection that uses the highpass subband as well seems to add more details (such as the separation of the upper detected region).

\section{CONCLUSION}

Analysis of fMRI data is a difficult task, mainly due to the low SNR of the data. In this paper, we have shown a link between two techniques that have - at first sight - nothing in common. However, two basic features of SPM, namely the Gaussian prefilter and the resels, can be related to the wavelet approach. Moreover, this correspondance allows us to compute an equivalent spline degree for which the lowpass part of wavelet analysis is essentially equivalent to SPM. 

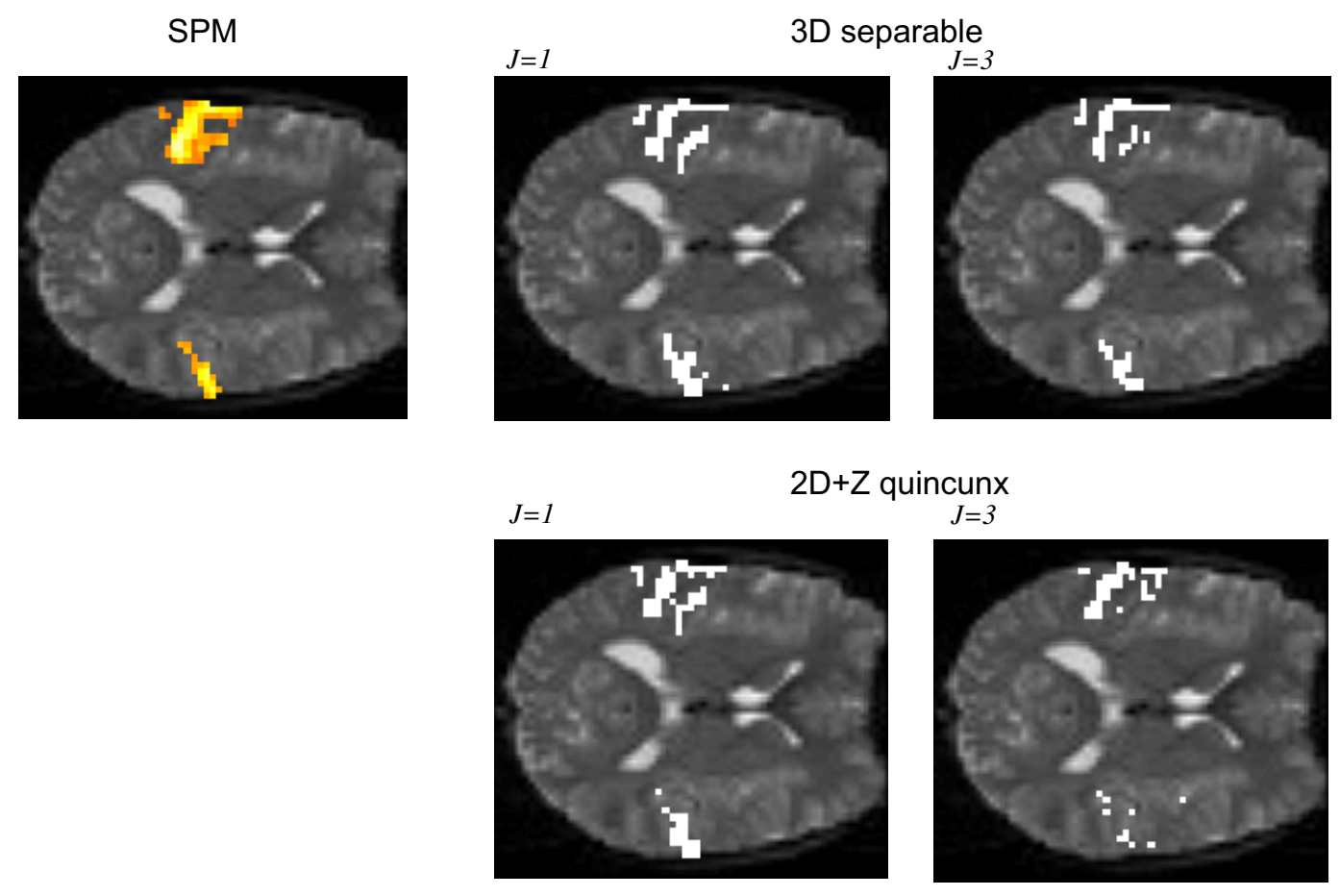

Figure 5. Example of activation in the auditory cortex. Left: SPM (FWHM $=6 \mathrm{~mm})$. Right: the wavelet approach. Equivalent degrees: 3D separable $J=1, \alpha=1.885 ; J=3, \alpha=1.198 ; 2 \mathrm{D}+\mathrm{Z}$ quincunx $J=1, \alpha_{q}=0.924$ and $\alpha_{z}=1.885$; $J=3, \alpha_{q}=0.466$ and $\alpha_{z}=1.198$.
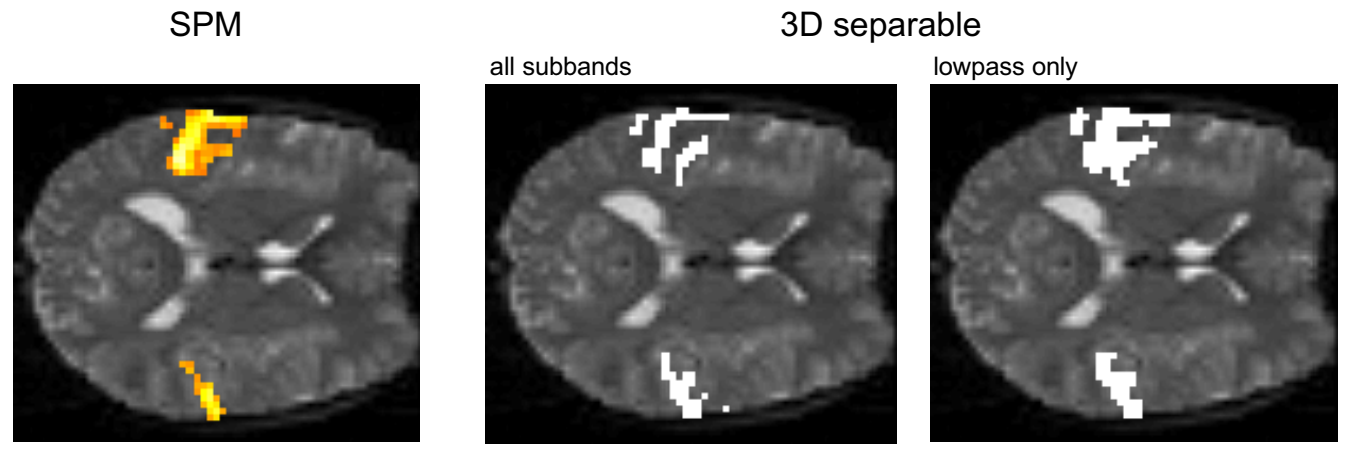

Figure 6. Example of activation in the auditory cortex. Left: SPM (FWHM=6mm). Middle: the wavelet approach (3D separable fractional-spline, $J=1$ ). Right: the same wavelet approach, but reconstructing only coefficients from the lowpass subband. 


\section{REFERENCES}

1. S. Ogawa, R. Menon, D. Tank, S. Kim, H. Merkle, J. Ellerman, and K. Ugurbil, "Functional brain mapping by blood oxygenation level-dependent constrast magnetic resonance imaging," Biophysical Journal 64, pp. 803-812, 1993.

2. M. Dagli, J. Ingeholm, and J. Haxby, "Localization of cardiac-induced signal change in fmri," NeuroImage 9, pp. 407-415, 1999.

3. E. Bullmore, M. Brammer, S. Rabe-Hesketh, V. Curtis, R. Morris, S. Williams, T. Sharma, and P. McGuire, "Methods for diagnosis and treatment of stimulus-correlated motion in generic brain activation studies using fmri," Human Brain Mapping 7, pp. 38-48, 1999.

4. R. Frackowiak, K. Friston, C. Frith, R. Dolan, and J. Mazziotta, Human Brain Function, Academic Press, 1997.

5. U. Ruttimann, M. Unser, R. Rawlings, D. Rio, N. Ramsey, V. Mattay, D. Hommer, J. Frank, and D. Weinberger, "Statistical analysis of functional mri data in the wavelet domain," IEEE Transactions on Medical Imaging 17(2), pp. 142-154, 1998.

6. M. Feilner, T. Blu, and M. Unser, "Analysis of fMRI data using spline wavelets," in Proceedings of the Tenth European Signal Processing Conference (EUSIPCO'00), IV, pp. 2013-2016, (Tampere, Finland), Sept. 2000.

7. M. Feilner, T. Blu, and M. Unser, "Statistical analysis of fMRI data using orthogonal filterbanks," in Proceedings of the SPIE Conference on Mathematical Imaging: Wavelet Applications in Signal and Image Processing VII, 3813, pp. 551-560, (Denver CO, USA), July 1999.

8. M. Feilner, T. Blu, and M. Unser, "Optimizing wavelets for the analysis of fMRI data," in Proceedings of the SPIE Conference on Mathematical Imaging: Wavelet Applications in Signal and Image Processing VIII, 4119, pp. 626-637, (San Diego CA, USA), July 2000.

9. K. J. Friston, A. P. Holmes, K. J. Worsley, J. P. Poline, C. D. Frith, and R. S. J. Fractowiak, "Statistical parametric maps in functional imaging: A general linear approach," Human Brain Mapping 2, pp. 189-210, 1995.

10. S. Mallat, "A theory for multiresolution signal decomposition: The wavelet decomposition," IEEE Trans. Pattern Anal. Mach. Intell. 11, pp. 674-693, 1989.

11. M. Unser and T. Blu, "Fractional splines and wavelets," SIAM Review 42, pp. 43-67, 2000.

12. T. Blu and M. Unser, "A complete family of scaling functions: The $(\alpha, \tau)$-fractional splines," in Proceedings of the Twenty-Eighth IEEE International Conference on Acoustics, Speech, and Signal Processing (ICASSP'03), IEEE, (Hong Kong), Apr. 2003.

13. J. Kovačević and M. Vetterli, "Nonseparable two- and three-dimensional wavelets," IEEE Transactions on Signal Processing 43(5), pp. 1269-1273, 1995.

14. M. Feilner, M. Jacob, and M. Unser, "Orthogonal quincunx wavelets with fractional orders," in Proceedings of the 2001 IEEE International Conference on Image Processing (ICIP'01), I, pp. 606-609, (Thessaloniki, Greece), October 7-10, 2001.

15. J. H. McClellan, "The design of two-dimensional digital filters by transformations," in Proc. 7th Annual Princeton Conf. Inf. Sciences and Systems, pp. 247-251, (Princeton, USA), 1973.

16. M. Unser, A. Aldroubi, and M. Eden, "On the asymptotic convergence of B-spline wavelets to Gabor functions," IEEE Transactions on Information Theory 38, pp. 864-872, March 1992.

17. G. Rees and K. Friston, "Single subject epoch (block) auditory fMRI activation data." http://www.fil.ion.ucl.ac.uk/spm/data/. 